Article

\title{
Cavity Quantum Electrodynamics of Continuously Monitored Bose-Condensed Atoms
}

\author{
Mark D. Lee and Janne Ruostekoski * \\ Mathematical Sciences, University of Southampton, Southampton SO17 1BJ, UK; \\ E-Mail: marklee1@gmail.com \\ * Author to whom correspondence should be addressed; E-Mail: janne@ @oton.ac.uk; \\ Tel.: +44-2380595142.
}

Academic Editor: Jonathan Goldwin and Duncan O'Dell

Received: 10 July 2015 / Accepted: 11 September 2015 / Published: 23 September 2015

\begin{abstract}
We study cavity quantum electrodynamics of Bose-condensed atoms that are subjected to continuous monitoring of the light leaking out of the cavity. Due to a given detection record of each stochastic realization, individual runs spontaneously break the symmetry of the spatial profile of the atom cloud and this symmetry can be restored by considering ensemble averages over many realizations. We show that the cavity optomechanical excitations of the condensate can be engineered to target specific collective modes. This is achieved by exploiting the spatial structure and symmetries of the collective modes and light fields. The cavity fields can be utilized both for strong driving of the collective modes and for their measurement. In the weak excitation limit the condensate-cavity system may be employed as a sensitive phonon detector which operates by counting photons outside the cavity that have been selectively scattered by desired phonons.
\end{abstract}

Keywords: ultracold atoms; cavity quantum electrodynamics; Bose-Einstein condensates; cavity optomechanics; phonon detection; continuous quantum measurement

PACS classifications: 42.50.Pq; 03.75.Gg; 42.50.Ct; 03.65.Ta 


\section{Introduction}

Cavity quantum electrodynamics (cQED) is a paradigm model of quantum optics [1]. In typical realizations a single atom interacts strongly with a single quantized light field. A large coupling coefficient $g$ of an atom to the cavity field, as compared with the cavity and spontaneous decay rates, then ensures a cooperative coupling regime. Recently it has also become possible to confine quantum degenerate atoms inside a single mode high-finesse optical cavity [2-8], representing an experimental milestone in extending quantum optical systems to the realm of quantum many-atom physics [9]. For example, atomic Bose-Einstein condensates (BECs) can be strongly coupled to an optical cavity field already at a single photon level.

The idea of optomechanics, the use of light forces to control and manipulate the quantum properties of mechanical oscillators, can be realized for the case of BECs in a cavity [4-6,10]. Here, typically, the motion of the condensate is employed as a mechanical device that is coupled to the cavity light field. In the case of a BEC, the oscillator is already in the ground state and there is no need for additional cooling. Optomechanics addresses the fundamental interest of oscillators operating in the quantum regime and is promising for highly sensitive measurements of weak forces at the quantum limit.

One of the central elements of quantum optics and quantum physics is the effect of quantum measurement on the evolution of a quantum system. A continuous measurement process forms a coupling of the quantum system to an environment. The resulting open quantum system dynamics is conditioned on the particular measurement record in each experimental run. In open interacting quantum many-body systems, the studies of continuous monitoring of the system evolution and the backaction of the quantum measurement process pose considerable challenges. For small quantum systems, the full quantum treatment of the measurement backaction may be incorporated in stochastic master equations and stochastic quantum trajectories of state vectors (quantum Monte Carlo wave functions) [11-13]. These approaches can produce a faithful representation of a possible measurement record for an individual experimental run, where the dynamics is conditioned on the stochastic measurement outcomes. For very large systems quantum trajectory simulations are not possible and there is a quest to develop approximate computationally efficient approaches. The motive for such developments is, e.g., the observation of the measurement backaction of the cavity output on the dynamics of the atoms in ultracold atom experiments [4,5]. Outside the ultracold regime, a continuous measurement process of the cavity output has been employed in the preparation of spin squeezed atomic ensembles [14].

Here we investigate the cavity optomechanics of a BEC, especially focusing on single stochastic realizations of the dynamics that are continuously monitored by detecting the light leaking out of the cavity. In the numerical simulations the BEC-cavity dynamics is integrated using classical stochastic measurement trajectories [15] that are based on stochastic differential equations (SDEs) for an approximate phase-space representation of a continuously monitored multimode system $[15,16]$. The advantage of the approximate treatment is that nonlinear evolution on a spatial grid of over a thousand points can be implemented and there is, e.g., no need for linearizing about a mean-field steady state solution [17-20].

Owing to the multimode nature of the BEC, the light not only excites the center-of-mass motion but also other collective modes of the condensate. The detection of light outside the cavity represents 
a combined excitation and measurement of the collective BEC modes. By exploiting the spatial structure and symmetries of the collective modes and light fields we can engineer the light excitation specifically to target certain modes. The driving of modes may also be achieved at higher light intensities but this is shown to considerably enhance the phase decoherence rate. The systems that are solely driven by the measurement of light outside the cavity are of special interest and represent a quantum measurement-induced spontaneous symmetry breaking: each stochastic realization exhibits a characteristic evolution of the density pattern, but ensemble-averaging over many runs restores the stationary unbroken spatial profile.

We show that, in the weak excitation limit, the selective coupling of the collective modes by the tailored BEC-cavity system may be employed as a sensitive phonon detector. The phonon detector is based on a photon counting of light leaking outside the cavity in which case the light that is not scattered by the phonons is suppressed by interference. The technique could potentially open the gate for a sensitive single phonon detector of BEC excitations that could measure statistical properties of the phonons, act as an accurate BEC thermometer, and prepare complex quantum states of phonons.

\section{Basic Model}

\subsection{Hamiltonian Formalism and Open System Dynamics}

In the following we introduce the formalism for the BEC-cavity system, illustrated in Figure 1, where the photons are leaking out of the cavity through the cavity mirrors. In the rotating wave approximation (and in the rotating frame of the pump field) we write the Hamiltonian for the closed system of two-level atoms and the cavity in the second-quantized form [21-23]

$$
H_{g e}=H_{A}+H_{C}+H_{C A},
$$

where the Hamiltonian terms for the atoms, $H_{A}$, for the cavity, $H_{C}$, and for the atom-cavity coupling, $H_{C A}$, are written as

$$
\begin{aligned}
H_{A} & =\int d x \hat{\Psi}_{g}^{\dagger}(x) H_{0}^{(g)} \hat{\Psi}_{g}(x)+\frac{U}{2} \int d x \hat{\Psi}_{g}^{\dagger}(x) \hat{\Psi}_{g}^{\dagger}(x) \hat{\Psi}_{g}(x) \hat{\Psi}_{g}(x) \\
& +\int d x \hat{\Psi}_{e}^{\dagger}(x)\left[H_{0}^{(e)}-\hbar \Delta_{p a}\right] \hat{\Psi}_{e}(x)-i \hbar \int d x\left[\hat{\Psi}_{g}^{\dagger}(x) h(x) \hat{\Psi}_{e}(x)-\hat{\Psi}_{e}^{\dagger}(x) h(x) \hat{\Psi}_{g}(x)\right] \\
H_{0}^{(j)} & =-\frac{\hbar^{2} \nabla^{2}}{2 m}+V^{(j)}(x) \\
H_{C A} & =-i \hbar \int d x \hat{\Psi}_{g}^{\dagger}(x) g(x) \hat{a}^{\dagger} \hat{\Psi}_{e}(x)+\text { H.c. } \\
H_{A} & =-\hbar \Delta_{p c} \hat{a}^{\dagger} \hat{a}+i \hbar \eta\left(\hat{a}^{\dagger}-\hat{a}\right) .
\end{aligned}
$$

Here $\omega_{c}$ and $\omega_{a}$ denote the cavity and atom resonance frequencies, respectively. An essentially arbitrary external trapping potential is denoted by $V(x)$. For the atomic fields $\hat{\Psi}_{g(e)}(x)$ annihilates an atom in the ground (excited) state at position $x$, while $\hat{a}$ annihilates a photon from the single cavity mode. The atom-cavity coupling is described by

$$
g(x)=g_{0} \sin (k x) .
$$


The cavity field is pumped along the cavity axis at a rate $\eta$ and via a transverse beam of profile $h(x)$. In practice, we consider situations where only one of the two driving mechanisms is used.

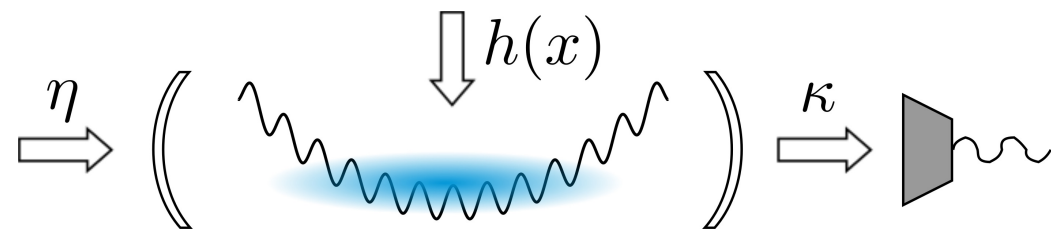

Figure 1. Schematic of the BEC-cavity system. The single-mode cavity can be pumped on axis at rate $\eta$, while the BEC inside the cavity can be pumped directly by a transverse beam of profile $h(x)$. The photons leaking from the cavity (at a rate $2 \kappa$ ) are continuously monitored. The atoms are subject to an external trapping potential $V(x)$ that is illustrated by the black line. Here the potential is shown mimicking a superposition of a periodic optical lattice and a harmonic trap, but generally the potential can take an essentially arbitrary form.

We assume that the atoms are tightly confined in a 1D cigar-shaped trap that is oriented along the cavity axis and neglect any density fluctuations of the atoms along the radial directions of the trap. The interatomic interactions for the ground state atoms are therefore represented by a 1D interaction strength $U=2 \hbar \omega_{\perp} a_{s}$, where $a_{s}$ denotes the $s$-wave scattering length and $\omega_{\perp}$ the radial trapping frequency for the atoms (the confinement perpendicular to the cavity field). The trapping potential along the axial direction is $V^{(j)}(x)$, where $j$ refers to either the excited- or the ground state atoms. The detunings between the pump frequency $\omega_{p}$ and the cavity and the atomic resonance frequencies are denoted by $\Delta_{p c}=\omega_{p}-\omega_{c}$ and $\Delta_{p a}=\omega_{p}-\omega_{a}$, respectively.

We will simplify the system description by considering a large detuning limit $\Delta_{c a} \gg \kappa$ (we also assume that $\Delta_{p a}$ is large so that the spontaneous emission to the modes other than the cavity mode may be ignored), where the excited state atomic field may be adiabatically eliminated. The dynamics may then be obtained from the effective Hamiltonian

$$
\begin{aligned}
H_{1} & =\int d x \hat{\Psi}^{\dagger}(x)\left\{H_{0}+\frac{\hbar}{\Delta_{p a}}\left[h^{2}(x)+g^{2}(x) \hat{a}^{\dagger} \hat{a}+h(x) g(x)\left(\hat{a}+\hat{a}^{\dagger}\right)\right]\right\} \hat{\Psi}(x) \\
& +\frac{U}{2} \int d x \hat{\Psi}^{\dagger}(x) \hat{\Psi}^{\dagger}(x) \hat{\Psi}(x) \hat{\Psi}(x)-\hbar \Delta_{p c} \hat{a}^{\dagger} \hat{a}-i \hbar \eta\left(\hat{a}-\hat{a}^{\dagger}\right) .
\end{aligned}
$$

Here $H_{0}$ now only refers to the ground state atoms and we drop the corresponding subscript.

So far, we have only described the closed system of the driven BEC and the cavity. The open-system description follows from the fact that cavity photons are leaking through the cavity mirrors at a rate $2 \mathrm{k}$. In order to analyze a continuous measurement process of photons outside the cavity, we will assume that all photons leaked out of the cavity are detected. The density operator $\rho_{\text {tot }}$ for the BEC-cavity system then evolves according to the master equation

$$
\frac{d \rho_{\mathrm{tot}}(t)}{d t}=-\frac{i}{\hbar}\left[H_{1}, \rho_{\mathrm{tot}}(t)\right]+\kappa \mathcal{L}[\hat{a}] \rho_{\mathrm{tot}}(t),
$$

where the superoperator $\mathcal{L}[\hat{O}]$ acting on $\hat{O}$ is defined by

$$
\mathcal{L}[\hat{O}] \rho \equiv\left(2 \hat{O} \rho \hat{O}^{\dagger}-\hat{O}^{\dagger} \hat{O} \rho-\rho \hat{O}^{\dagger} \hat{O}\right)
$$


The next level of simplification for the dynamics can be obtained by also adiabatically eliminating the cavity light mode. This can be done in the bad cavity limit $\kappa \gg N g_{0}^{2} / \Delta_{p a}$. The elimination is performed for the open system dynamics by means of unitary transformations, but we give here only a simple heuristic explanation of the derivation (for a more rigorous elimination, the reader is referred to [15]). The equation of motion for $\hat{a}$ reads

$$
\frac{d \hat{a}}{d t}=\left(i\left[\Delta_{p c}-\frac{1}{\Delta_{p a}} \int \hat{\Psi}^{\dagger}(x) \hat{\Psi}(x) g^{2}(x) d x\right]-\kappa\right) \hat{a}+\eta-\frac{i}{\Delta_{p a}} \int \hat{\Psi}^{\dagger}(x) \hat{\Psi}(x) h(x) g(x) d x .
$$

The cavity field is then eliminated by setting

$$
\hat{a}=\frac{1}{\mathrm{~K}-i \tilde{\Delta}_{p c}}\left(\eta-\frac{i}{\Delta_{p a}} \int \hat{\Psi}^{\dagger}(x) \hat{\Psi}(x) h(x) g(x) d x\right), \quad \tilde{\Delta}_{p c} \equiv \Delta_{p c}-\frac{1}{\Delta_{p a}} \int \hat{\Psi}^{\dagger}(x) \hat{\Psi}(x) g^{2}(x) d x,
$$

We expand the denominator in terms of the small parameter $\tilde{\Delta}_{p c} / \kappa\left(\right.$ valid when $\left.\kappa \gg \Delta_{p c}, N g_{0}^{2} / \Delta_{p a}\right)$, leading to

$$
\hat{a} \simeq \frac{1}{\kappa}\left(\eta-\frac{i}{\Delta_{p a}} \int \hat{\Psi}^{\dagger}(x) \hat{\Psi}(x) h(x) g(x) d x\right)\left[1+i \frac{\tilde{\Delta}_{p c}}{\mathrm{\kappa}}+O\left(\frac{\tilde{\Delta}_{p c}^{2}}{\mathrm{~K}^{2}}\right)\right] .
$$

For the case that we only pump transversely (with a beam profile $h(x)$ ) we set $\eta=0$. Then

$$
\hat{a} \simeq-i \frac{\hat{Y}}{\kappa}\left(1+i \frac{\Delta_{p c}}{\mathrm{\kappa}}-i \frac{\hat{X}}{\mathrm{\kappa}}\right)
$$

where $\hat{Y}$ represents the off-resonant excitation of the atoms via the transverse pump beam and $\hat{X}$ excitation via the cavity field

$$
\hat{Y} \equiv \int d x \frac{h(x) g(x)}{\Delta_{p a}} \hat{\Psi}^{\dagger}(x) \hat{\Psi}(x), \quad \hat{X} \equiv \int d x \frac{g^{2}(x)}{\Delta_{p a}} \hat{\Psi}^{\dagger}(x) \hat{\Psi}(x) .
$$

Eliminating the light field in the lowest order approximation (only keeping the first term in Equation (13)) then results in an effective Hamiltonian and the master equation

$$
\begin{gathered}
H_{\text {tra }}=\int d x \hat{\Psi}^{\dagger}(x)\left[H_{0}+\frac{U}{2} \hat{\Psi}^{\dagger}(x) \hat{\Psi}(x)+\hbar \frac{h^{2}(x)}{\Delta_{p a}}\right] \hat{\Psi}(x), \\
\frac{d \rho_{a}(t)}{d t}=-\frac{i}{\hbar}\left[H_{\text {tra }}, \rho_{a}(t)\right]+\frac{1}{\kappa} \mathcal{L}[\hat{Y}] \rho,
\end{gathered}
$$

As the light field is eliminated, the measurement observable now depends solely on atomic operators [15]. In particular, the measurement operator involves an integral over a non-uniform multimode quantum field $\hat{\Psi}(x)$ combined with a spatially varying pump profile and the cavity coupling strength. The rate of measurement is given by

$$
r_{\text {meas }}(t)=\frac{2}{\kappa}\langle\hat{Y} \hat{Y}\rangle=2 \kappa n,
$$

which we have expressed in terms of the number of photons in the cavity $n=\left\langle\hat{a}^{\dagger} \hat{a}\right\rangle=\left\langle(\hat{Y} / \kappa)^{2}\right\rangle$. Here all cavity photons appear from interactions of the transverse beam with atoms and the rate of measurement events that affect the atoms $r_{\text {meas }}$ is therefore simply that of the number of photons leaving the cavity. 
For the case that the cavity mode is driven axially and there is no transverse pumping of the atoms $(h(x)=0$ ), eliminating the cavity field operator leads, in the lowest order in our expansion parameter, to the Hamiltonian and master equation for the atoms

$$
\begin{gathered}
H_{\mathrm{axi}}=\int d x \hat{\Psi}^{\dagger}(x)\left[H_{0}+\frac{U}{2} \hat{\Psi}^{\dagger}(x) \hat{\Psi}(x)+\hbar \frac{|\eta|^{2}}{\mathrm{\kappa}^{2}} \frac{g^{2}(x)}{\Delta_{p a}}\right] \hat{\Psi}(x), \\
\frac{d \rho_{a}(t)}{d t}=-\frac{i}{\hbar}\left[H_{\mathrm{axi}}, \rho_{a}(t)\right]+\frac{|\eta|^{2}}{\mathrm{\kappa}^{3}} \mathcal{L}[\hat{X}] \rho_{a}(t) .
\end{gathered}
$$

and the rate of scattered photons counted by the measurement apparatus is

$$
r_{\text {meas }}(t)=\frac{2|\eta|^{2}}{\kappa^{3}}\langle\hat{X} \hat{X}\rangle \text {. }
$$

The master equations, i.e., Equations (8), (16) and (19), represent an ensemble average over a large number of measurement realizations and are not conditioned on any particular measurement record. They do not incorporate information about individual stochastic runs. A common approach to describe the backaction of a continuous quantum measurement is to unravel the master equation into quantum trajectories of the state vectors [11-13,24]. A full quantum treatment of quantum trajectory simulations is computationally demanding in large systems and in the following we will develop approximate methods for individual stochastic runs.

\subsection{Phase-Space and Stochastic Descriptions}

Typical BEC-cavity systems may consist of spatial atom dynamics that require well over 100 modes for an accurate description. On the other hand, the atom number in the cavity may commonly vary between $10^{3}$ and $10^{6}$. This is a significantly larger system than what the full quantum description would allow in a numerical simulation. Here we introduce an approximate computationally feasible approach that is based on classical phase-space methods. The state of the multimode atom-light system can be expressed by the Wigner function $W\left(\alpha, \alpha^{*},\left\{\psi, \psi^{*}\right\}\right)$, where $\alpha$ is the classical variable associated with $\hat{a}$, and $\psi$ is a classical field representation of the field operator $\hat{\Psi}$ that is stochastically sampled from an ensemble of Wigner distributed classical fields. The full quantum dynamics of the master equation can be mapped to a phase-space dynamics of the Wigner function using standard techniques of quantum optics [22,25]. Neither the master equation for the density matrix nor the phase-space dynamics for the Wigner function are conditioned on any particular measurement record, but represent an ensemble average over a large number of measurement realizations. In order to describe a single experimental run of a continuously monitored BEC-cavity system, where the photons leaking out of the cavity are measured and the state of the system is determined by the detection record, we need to have an alternative representation to the ensemble-averaged ones. We will therefore derive classical stochastic measurement trajectories from an approximate description of the phase-space dynamics. Each such a trajectory is a faithful representation of a single experimental run where the dynamical noise of the equations is associated with the measurement noise and the detection record of the output light from the cavity.

The equation of motion for the Wigner function of the BEC-cavity system may be derived from the master Equation (8) via the operator correspondences [22,25] similar to

$$
\hat{\Psi} \rho \leftrightarrow\left(\psi+\frac{1}{2} \frac{\delta}{\delta \psi^{*}}\right) W\left(\alpha, \alpha^{*},\left\{\psi, \psi^{*}\right\}\right) .
$$


The substitution of the operator correspondences to the master equation leads to a Fokker-Planck equation (FPE) for the Wigner function in the limit of weak quantum fluctuations [15]. Specifically, for the interatom interactions we consider the limit the atom number $N \rightarrow \infty$, while keeping $C=N U$ constant. Analogously, for the atom-photon interaction terms we take the limit where the number of cavity photons $n \rightarrow \infty$ while the maximum atom-photon interaction energies $\chi=\hbar\left(g_{0}^{2} / \Delta_{p a}\right) n$ and $\chi_{h}=\hbar\left(h_{0} g_{0} / \Delta_{p a}\right) \sqrt{n}$ remain constant (note that the transverse pump scales as $h_{0} \propto \sqrt{n}$ ). The interatomic interaction limit can be related to the 1D Tonks parameter [26] $\gamma=m U /\left(\hbar^{2} \rho_{1 D}\right) \gg 1$, where $\rho_{1 D}$ is the one-dimensional atom density, indicating that the expansion is strictly valid in the regime of a weakly interacting bosonic gas (when the Bogoliubov approximation becomes accurate for the ground state atoms), although especially in 1D systems short-time behavior can be qualitatively described even for more strongly fluctuating cases [27]. The classical approach can also be significantly more accurate in estimating the dynamics of the measured observable even deep in the quantum regime [16]. The Tonks parameter measures the ratio of the nonlinear $s$-wave interaction to kinetic energies for atoms spaced at the mean interatomic distance and the expansion also implies that the number of atoms found within a healing length $\xi$ is $N_{\xi} \simeq 1 / \sqrt{2 \gamma} \gg 1$.

In the limit of weak quantum fluctuations the FPE for the approximate BEC-cavity system then reads [15]

$$
\frac{\partial}{\partial t} W=-\sum_{i} \frac{\partial}{\partial q_{i}} A_{i} W+\frac{\kappa}{2} \frac{\partial^{2}}{\partial \alpha \partial \alpha^{*}} W+\frac{\kappa}{2} \frac{\partial^{2}}{\partial \alpha^{*} \partial \alpha} W,
$$

where the index $q_{i}$ runs over the set $\left\{\alpha, \alpha^{*}, \psi(x), \psi^{*}(x)\right\}$. The nonlinear atom-light dynamics is incorporated in the drift term elements $A_{i}$ that arise from the unitary Hamiltonian Equation (7)

$$
\begin{aligned}
& A_{\alpha}=-\eta+i \int \frac{h(x) g(x)}{\Delta_{p a}}|\psi(x)|^{2} d x+\left(\kappa-i \Delta_{p c}+i \int \frac{g^{2}(x)}{\Delta_{p a}}|\psi(x)|^{2} d x\right) \alpha \\
& A_{\psi(x)}=\frac{i}{\hbar} \int d x\left(H_{0}+U|\psi(x)|^{2}+\hbar\left[\frac{h^{2}(x)}{\Delta_{p a}}+\frac{g^{2}(x)|\alpha|^{2}}{\Delta_{p a}}+\frac{h(x) g(x)}{\Delta_{p a}}\left(\alpha+\alpha^{*}\right)\right]\right) \psi(x)
\end{aligned}
$$

The last two terms in Equation (22) can be physically associated with the backaction of the continuously measured light leaking out of the cavity, and they form the diffusion part of the equation.

In deriving Equation (22) we have neglected the terms containing higher derivatives than the second order ones by taking the weak fluctuation limit. The advantage of expressing the dynamics as a FPE follows from their mathematical correspondence to systems of SDEs [25,28]. Besides the computational simplicity of SDEs as compared with Equation (22), we can now also obtain a stochastic description for single realizations of a continuous measurement process. On the other hand, the corresponding FPE corresponds to an ensemble average over all possible measurement outcomes that has discarded the individual measurement records.

The derivation of an FPE is reminiscent of dropping the triple derivative terms that arise from the $s$-wave interactions in the truncated Wigner approximation [29] that has been actively utilized in the studies of bosonic atom dynamics in closed systems [30-36] (for a recent work on using truncated Wigner approximation in cold atoms see, e.g., [37-43]) as well as when incorporating three-body losses of atoms in a trap $[36,44]$.

An FPE can be mathematically mapped to Ito SDEs. For the cavity system we can express Equation (22) as coupled SDEs for the stochastic light and atomic amplitudes $\alpha(t)$ and $\psi(x, t)$, 
respectively. These equations can be numerically integrated even for large systems and they represent an unraveling of the FPE into classical stochastic measurement trajectories [15]. The trajectories describe individual continuous measurement processes where the dynamics is conditioned on the detection record. Here we, however, focus on the specific limit of a bad cavity $\mathrm{k} \gg N g_{0}^{2} / \Delta_{p a}$, when the cavity light field can be adiabatically eliminated from the dynamics. We consider the transversely pumped case of Equation (16). Having eliminated the cavity field we can now use a Wigner representation in terms solely of the atomic variables $W\left(\left\{\psi(x), \psi^{*}(x)\right\}\right)$. The approximate FPE can be derived in the case of weak quantum fluctuations using the same principles as Equation (22). We find

$$
\frac{\partial}{\partial t} W=\left.\frac{\partial}{\partial t} W\right|_{\text {Ham }}+\left.\frac{\partial}{\partial t} W\right|_{\text {meas }}
$$

where the first term corresponds to that from the Hamiltonian evolution governed by Equation (16)

$$
\left.\frac{\partial}{\partial t} W\right|_{\text {Ham }}=\frac{i}{\hbar} \int d x \frac{\partial}{\partial \psi}\left[H_{0}+U|\psi(x)|^{2}+\hbar \frac{h^{2}(x)}{\Delta_{p a}}\right] \psi(x) W+\text { c.c. },
$$

while the measurement term has the form

$$
\begin{aligned}
\frac{\partial}{\partial t} W & =\frac{1}{\mathrm{\kappa}} \int d x \frac{h^{2}(x) g^{2}(x)}{\Delta_{p a}^{2}} \frac{\delta}{\delta \psi(x)} \psi(x) W \\
& +\frac{1}{\mathrm{\kappa}} \int d x d x^{\prime} \frac{h(x) g(x)}{\Delta_{p a}(x)} \frac{h\left(x^{\prime}\right) g\left(x^{\prime}\right)}{\Delta_{p a}\left(x^{\prime}\right)}\left(\frac{\delta^{2}}{\delta \psi(x) \delta \psi^{*}\left(x^{\prime}\right)} \psi(x) \psi^{*}\left(x^{\prime}\right)-\frac{\delta^{2}}{\delta \psi(x) \delta \psi\left(x^{\prime}\right)} \psi(x) \psi\left(x^{\prime}\right)\right) W \\
& + \text { c.c. }
\end{aligned}
$$

The diffusion matrix of the FPE for the part that does not involve measurements vanishes identically. Symmetrically-ordered expectation values $\langle\cdots\rangle_{W}$ for the atomic fields are obtained with respect to the quasidistribution function $W\left(\left\{\psi(x), \psi^{*}(x)\right\}\right)$

$$
\left\langle\psi^{*}\left(x_{1}\right) \cdots \psi^{*}\left(x_{k}\right) \psi\left(x_{k+1}\right) \cdots \psi\left(x_{l}\right)\right\rangle_{W}=\int d^{2} \psi W\left(\left\{\psi, \psi^{*}\right\}\right) \psi^{*}\left(x_{1}\right) \cdots \psi^{*}\left(x_{k}\right) \psi\left(x_{k+1}\right) \cdots \psi\left(x_{l}\right) .
$$

The FPE can then be unraveled into classical trajectories for the stochastic field $\psi(x)$ obeying

$$
d \psi(x)=\left\{\frac{-i}{\hbar}\left[H_{0}+U|\psi(x)|^{2}\right]-i \frac{h^{2}(x)}{\Delta_{p a}(x)}-\frac{1}{\kappa} \frac{h^{2}(x) g^{2}(x)}{\Delta_{p a}^{2}(x)}\right\} \psi(x) d t-i \sqrt{\frac{2}{\kappa}} \frac{h(x) g(x)}{\Delta_{p a}(x)} \psi(x) d W,
$$

where $d W$ denotes a Wiener increment with $\langle d W\rangle=0,\left\langle d W^{2}\right\rangle=d t$.

At first glance, the last term proportional to $d t$ would appear to give rise to non-unitary evolution. However, this term counteracts the non-unitary evolution introduced by the term proportional to $d W$, and the total atom number is in fact conserved by Equation (29). These two terms, which are $\propto h(x) g(x)$, represent the effect of the light detection record on the atoms. Of those terms which explicitly lead to unitary evolution, the one that is proportional to $h^{2}(x)$ describes the light shift due to the transverse beam, while the remainder describe the evolution of the BEC that would occur in the absence of the cavity mode. 
The stochastic noise in Equation (29) is a physical consequence of the backaction of a continuous quantum measurement process of the light that has leaked out of the cavity. In this classical approximation to a single experimental run the dynamics is conditioned on the detection record. The noise term in the SDE Equation (29) directly results from the diffusion term in the corresponding FPE. The ensemble average of the dynamics over many stochastic realizations generates the unconditioned expectation values Equation (28). Different multimode treatments of continuously measured systems, which are based on alternative phase-space approaches and are also suitable for cavity systems, were developed in $[45,46]$. The method also has similarities to numerical approaches to "stochastic electrodynamics", see for instance [47].

In the stochastic representation the initial conditions $W\left(\left\{\psi, \psi^{*}\right\}, t=0\right)$ correspond to a (Wigner-distributed) classical probability distribution for the initial state. Thermal and quantum fluctuations may be included in the initial state of $\psi$ within the constraint that the corresponding $W\left(\left\{\psi, \psi^{*}\right\}, t=0\right)$ remains positive [48]. This still allows notable quantum fluctuations, such as mode and spin squeezing, to be incorporated. In practical situations, for an accurate modeling of short-time dynamics it is often necessary to sample the initial conditions $\psi(x, t=0)$ of individual stochastic realizations from $W\left(\left\{\psi, \psi^{*}\right\}, t=0\right)$ using many-body theories that sufficiently well reproduce the correct quantum statistical correlations for an initially stable equilibrium configuration of the system. For simplicity, we consider the initial configuration of the atoms in the ground state inside the cavity in the absence of the light field. The general idea is to represent the many-body initial state in terms of some non-interacting quasiparticles, with annihilation and creation operators $\hat{\beta}_{j}$ and $\hat{\beta}_{j}^{\dagger}$ respectively, that satisfy the ideal gas phonon statistics

$$
\left\langle\hat{\beta}_{j}^{\dagger} \hat{\beta}_{j}\right\rangle=\bar{n}_{j}=\frac{1}{\exp \left(\epsilon_{j} / k_{B} T\right)-1} .
$$

We then replace the quantum operators $\left(\hat{\beta}_{j}, \hat{\beta}_{j}^{\dagger}\right)$ by the complex stochastic variables $\left(\beta_{j}, \beta_{j}^{*}\right)$, obtained by sampling the corresponding Wigner distribution of the quasiparticles. The operators $\left(\hat{\beta}_{j}, \hat{\beta}_{j}^{\dagger}\right)$ behave as a collection of ideal harmonic oscillators whose Wigner distribution in a thermal bath reads [25]

$$
W\left(\beta_{j}, \beta_{j}^{*}\right)=\frac{2}{\pi} \tanh \left(\xi_{j}\right) \exp \left[-2\left|\beta_{j}\right|^{2} \tanh \left(\xi_{j}\right)\right],
$$

where $\xi_{j} \equiv \epsilon_{j} / 2 k_{B} T$. The nonvanishing contribution to the width $\bar{n}_{j}+\frac{1}{2}$ of the Gaussian distribution at $T=0$ for each mode represents the quantum noise. Since the Wigner function returns symmetrically ordered expectation values, we have

$$
\left\langle\beta_{j}^{*} \beta_{j}\right\rangle_{W}=\int d^{2} \beta_{j} W\left(\beta_{j}, \beta_{j}^{*}\right)\left|\beta_{j}\right|^{2}=\bar{n}_{j}+\frac{1}{2},
$$

and similarly $\left\langle\beta_{j}\right\rangle_{W}=\left\langle\beta_{j}^{*}\right\rangle_{W}=\left\langle\beta_{j}^{2}\right\rangle_{W}=0$.

In the Bogoliubov approximation we introduce the quasiparticles by expanding the field operator $\hat{\psi}(x, t=0)$ in terms of the BEC ground state amplitude $\hat{\beta}_{0} \psi_{0}$, with $\left\langle\hat{\beta}_{0}^{\dagger} \hat{\beta}_{0}\right\rangle=N_{0}$ (here $N_{0}$ denotes the $\mathrm{BEC}$ atom number that excludes the depleted atoms in the excited states), and the excited states [32]

$$
\hat{\psi}(x)=\psi_{0}(x) \hat{\beta}_{0}+\sum_{j>0}\left[u_{j}(x) \hat{\beta}_{j}-v_{j}^{*}(x) \hat{\beta}_{j}^{\dagger}\right] .
$$


The ground state BEC solution $\psi_{0}\left(\int d x\left|\psi_{0}(x)\right|^{2}=1\right)$, the quasiparticle mode functions $u_{j}(x)$ and $v_{j}(x)(j>0)$, and the corresponding eigenenergies $\epsilon_{j}$, can be solved numerically. In a more strongly fluctuating case the quasiparticle modes and the ground state condensate profile may be solved self-consistently using the Hartree-Fock-Bogoliubov theory [38,49]. A strongly confined 1D system may also exhibit enhanced phase fluctuations that can be incorporated using a quasicondensate representation [34]. Finally, the initial state $\psi(x, t=0)$ for the stochastic simulations that synthesizes the appropriate statistics may then be constructed from $\hat{\psi}(x, t=0)$ in Equation (33) by replacing $\left(\hat{\beta}_{j}, \hat{\beta}_{j}^{\dagger}\right)$ by the stochastically sampled $\left(\beta_{j}, \beta_{j}^{*}\right)$.

\section{Numerical Results}

\subsection{Cavity Optomechanical System}

The coupling of a BEC to the cavity light mode and the continuous measurement of light leaking out of the cavity generate a mechanical response on the atoms. In the field of cavity optomechanics the coupling of a variety of mechanical oscillators to the cavity field has been actively investigated in recent years [50-53]. Nanomechanical resonators have been cooled to the quantum regime [54-56], with potential applications, e.g., to quantum sensing. A BEC in a cavity already forms the ground state where the atomic motion can be coupled to the cavity mode [4-6,10,57].

The coupling of the cavity field to intrinsic collective excitations of a BEC was studied using classical measurement trajectories in [15]. Here we expand those studies and focus in particular on single trajectories that are conditioned on the measurement record on the light outside the cavity. We show how the system can be engineered in such a way that the light excitation specifically targets certain modes and how varying the pump power affects the resulting dynamics. Here we also demonstrate how in the weak excitation limit the condensate-cavity system may be employed as a sensitive phonon detector that operates by counting photons outside the cavity that have been selectively scattered by desired phonons.

The continuous measurements generate optomechanical dynamics of a BEC. Owing to the multimode nature of the BEC, the number of internal dynamical degrees of freedom of the atoms is large, involving many collective excitation modes. It is especially interesting to consider situations where the collective excitations of the BEC are solely the consequence of the conditioned measurement record of a single stochastic realization. In such cases the individual trajectories may display a notable variety of different dynamics, but ensemble-averaging over a large number of realizations then cancels out any overall motion in the atomic density.

We consider regimes where the excitations of the BEC are not small and they also exhibit a complex spatial structure, such that standard treatments of the linear optomechanical regime are insufficient. The phonon excitations are a characteristic signature of the BEC nonlinearity. In general, detection of the light leaking out of the cavity represents a combined measurement and excitation of several of the interacting collective modes of the BEC. However, we demonstrate examples of how the measurement can be tailored to preferentially excite and detect selected intrinsic excitations. 
For simplicity, we consider a BEC confined in a harmonic well inside an optical cavity and set $V(x)=$ $m \omega^{2} x^{2} / 2$, with corresponding length scale $x_{0}=\sqrt{\hbar /(m \omega)}$ and time scale $t_{0}=1 / \omega$. The wavelength of the cavity is assumed to be of the same order as the size of the BEC. This system also represents the translationally invariant case of many BECs in a periodic potential with negligible tunneling, with each BEC coupled identically.

In the simulation examples we consider the limit of weak quantum fluctuations and the limit where the light field can be adiabatically eliminated ( $n \gg 1$ and $\left.N g_{0}^{2} / \Delta_{p a} \ll \kappa\right)$, corresponding to Equation (29). The SDE Equation (29) is numerically integrated using a semi-implicit Milstein algorithm [58] on a spatial grid of 1024 points. In the simulations the atoms are pumped at the cavity mode resonance transversely with a uniform driving field $h(x)=h_{0}$ and with zero axial pumping. We set $N U \approx 64 \hbar \omega x_{0}$ and $h_{0}^{2} g_{0}^{2} / \mathrm{k} \Delta_{p a}^{2} \approx 0.042 \omega$. For ${ }^{87} \mathrm{Rb}$ we have $a_{s} \simeq 5 \mathrm{~nm}$. Then, for instance, setting $x_{0} \simeq 300 \mathrm{~nm}$ and $\omega / \omega_{\perp} \simeq 0.1$ yields $N \simeq 190$. The quantum and thermal fluctuations in the initial state are assumed to be negligible (low temperature and large atom number, which for the given nonlinearity translates to $N \gg 14)$. The large $N$ limit also guarantees a sufficiently fast detection rate and the validity of the adiabatic expansion $\int\left(g^{2}(x) / \Delta_{p a}\right)|\psi(x)|^{2} d x \ll \kappa$.

\subsection{Selective Phonon Excitations and Their Measurements}

In Figure 2 we show the evolution of individual classical measurement trajectories when the dynamics conditioned on the detection record excites the center-of-mass motion of the BEC. This induced optomechanical coupling to a BEC corresponds to the excitation of the lowest energy collective BEC mode (the Kohn mode). The cavity wavelength maximizes the overlap integral $O_{1}$ of Equation (43) in the Appendix for the Kohn mode, as illustrated in Figure 2e.

In the specific numerical examples studied, the sole dynamical contribution from the cavity mode is due to measurement backaction. The BEC is initially in the ground state and all dynamics is therefore purely measurement induced and any differences between the trajectories arise from the stochastic detection record. The presented simulation results therefore also indicate a spontaneous measurement-induced symmetry breaking in the dynamics of the atoms.

In Figure 2 the center-of-mass oscillations are notable. The individual stochastic runs also considerably differ from each other. Collective mode amplitudes and phases also vary between realizations. The oscillations are revealed as pulses in the rate of photon measurements, since the number of photons pumped into the cavity mode depends upon the time varying overlap of the stochastic field $\psi(x, t)$ with the cavity mode $g(x)$.

While individual measurement trajectories show distinct effects of measurement backaction, an ensemble average over a large number of independent trajectories restores the initial unbroken symmetry, as shown in Figure 3. The ensemble averaged results reproduce the dynamics from the unconditioned FPE Equation (25), and such dynamics show no overall motion of the condensate density. The unconditioned dynamics are not trivial, however, and the dissipation caused by the open nature of the system leads to decoherence of the condensate (Figure 4c). 

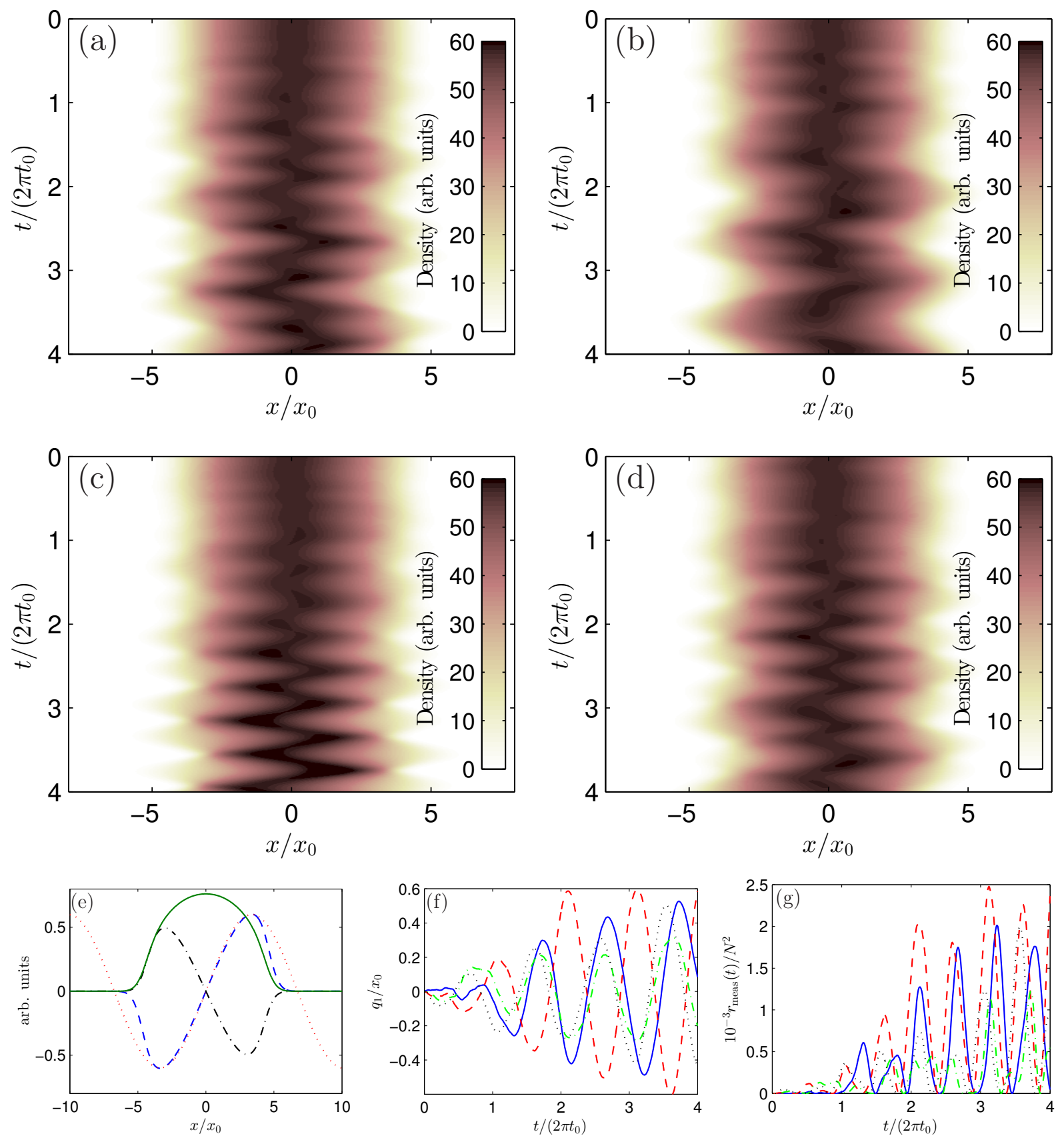

Figure 2. A selective excitation of collective modes of a BEC as a result of quantum measurement backaction. (a-d) The stochastic field density $|\psi(x, t)|^{2}$ as a function of time for four different single realizations of measurement trajectories in which the Kohn mode is excited. The transverse pump beam is applied at $t=0$ and remains at a constant strength throughout the simulation. (e) Comparison of the shapes of the Kohn mode quasiparticle functions $u_{1}(x)$ (blue, dashed) and $v_{1}(x)$ (black, dashed) with the cavity mode function $g(x)$ (red, dotted), and the initial stochastic field for the condensate $\psi(x)$ (green, solid), for the case where the overlap of the cavity mode and the Kohn mode is maximal. Subfigure (e) reproduced from [15]. The differing quantities have been scaled into arbitrary units to enable a comparison of their functional form. (f-g) The center-of-mass position $q_{1}(t)=\int x|\psi(x, t)|^{2} d x$ and measurement rate of photons $r_{\text {meas }}(t)$ corresponding to the single realizations illustrated in (a) (blue, solid), (b) (red, dashed), (c) (green, dot-dashed) and (d) (black, dotted). 

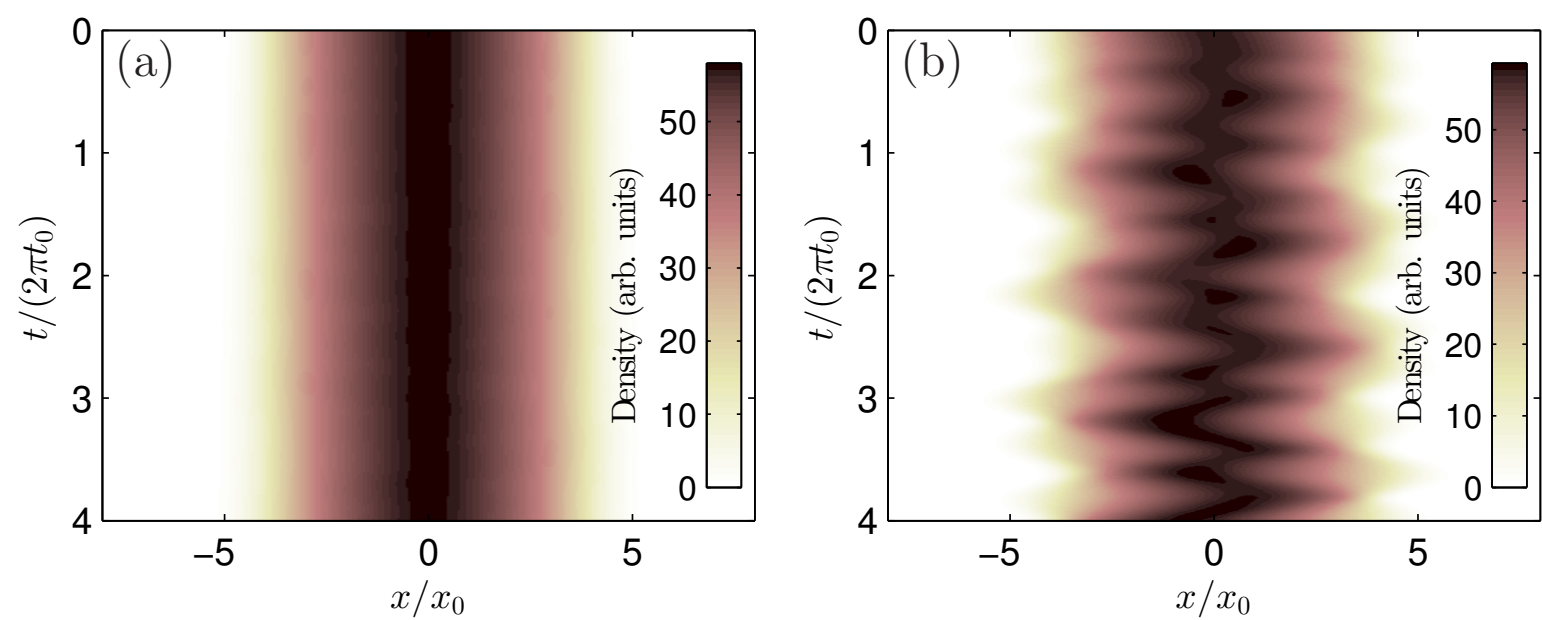

Figure 3. Measurement-induced symmetry breaking of a spatial condensate pattern and the restored symmetry in the ensemble average over many individual realizations of classical stochastic measurement trajectories. (a) Ensemble averaged stochastic field density $|\psi(x, t)|^{2}$ of the BEC response unconditioned on any particular measurement trajectory, formed by ensemble averaging 400 single trajectories. (b) A single trajectory that exhibits a typical measurement-induced spatial symmetry breaking, shown here for comparison.
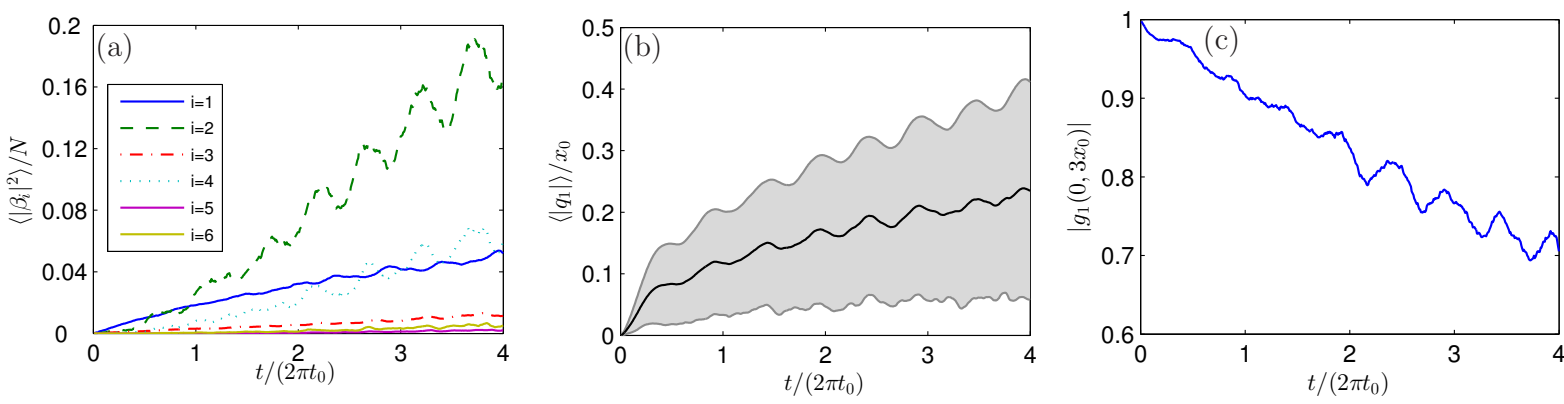

Figure 4. (a) Average response of a single trajectory, decomposed into Bogoliubov linearized collective excitations, mode occupation numbers $\left\langle\left|\beta_{i}\right|^{2}\right\rangle$ are shown for the lowest six modes. (b) Average center-of-mass displacement $q_{1}(t)=\int x|\psi(x, t)|^{2} d x$ for a single measurement conditioned trajectory. Note the absolute value must be taken since the measurement backaction generates oscillations with a random phase for different realizations. (c) Dissipation induced loss of coherence in the ensemble averaged unconditioned evolution corresponding to that shown in Figure 3a. The figure shows $\left|g_{1}\left(x, x^{\prime}\right)\right| \equiv\left|\left\langle\hat{\Psi}^{\dagger}(x) \hat{\Psi}\left(x^{\prime}\right)\right\rangle\right|$ between $x=0$ and $x^{\prime}=3 x_{0}$. The initial condensate is phase coherent with $\left|g_{1}\left(x, x^{\prime}\right)\right|=1$. Subfigure (c) reproduced from [15].

In contrast to unconditioned ensemble dynamics, Figure $4 a, b$ presents the average response of a single measurement conditioned trajectory. As anticipated, from a Bogoliubov mode decomposition it can be seen that the center-of-mass motion is largely due to an excitation of the Kohn mode. However, the multimode nature of the system is illustrated by the number of other modes that are also excited. Notably, two even-symmetry modes respond strongly to the odd symmetry $g(x)$, a situation made possible once the center-of-mass motion breaks the even symmetry of $\psi(x, t)$. 
Both the measurement-induced mechanical motion of single trajectories and the decoherence of the unconditioned ensemble averaged dynamics are sensitive to the transverse pump power, as illustrated in Figure 5. For larger pump powers the induced mechanical motion becomes of larger amplitude but at a cost of a considerable complication in the motion, as the multimode nature of the condensate couples several intrinsic modes. Similarly, increasing the pump power greatly enhances the rate of decoherence of the condensate in the unconditioned dynamics. The induced phase decoherence of the BEC can be illustrated in terms of the density and the phase of the atoms $\psi(x)=f(x) \exp (i \Phi(x))$, where $f^{2}(x)$ corresponds to the density and $\Phi(x)$ the phase. We can then obtain an alternative representation of FPE in terms of the Wigner function $W(\{f(x), \Phi(x)\})$. The stochastic measurement part of the corresponding SDE reads

$$
\left.\frac{d f(x)}{d t}\right|_{\text {meas. }}=0,\left.\quad d \Phi(x)\right|_{\text {meas. }}=\sqrt{\frac{2}{\kappa}} \frac{g(x) h(x)}{\Delta_{p a}(x)} d W .
$$
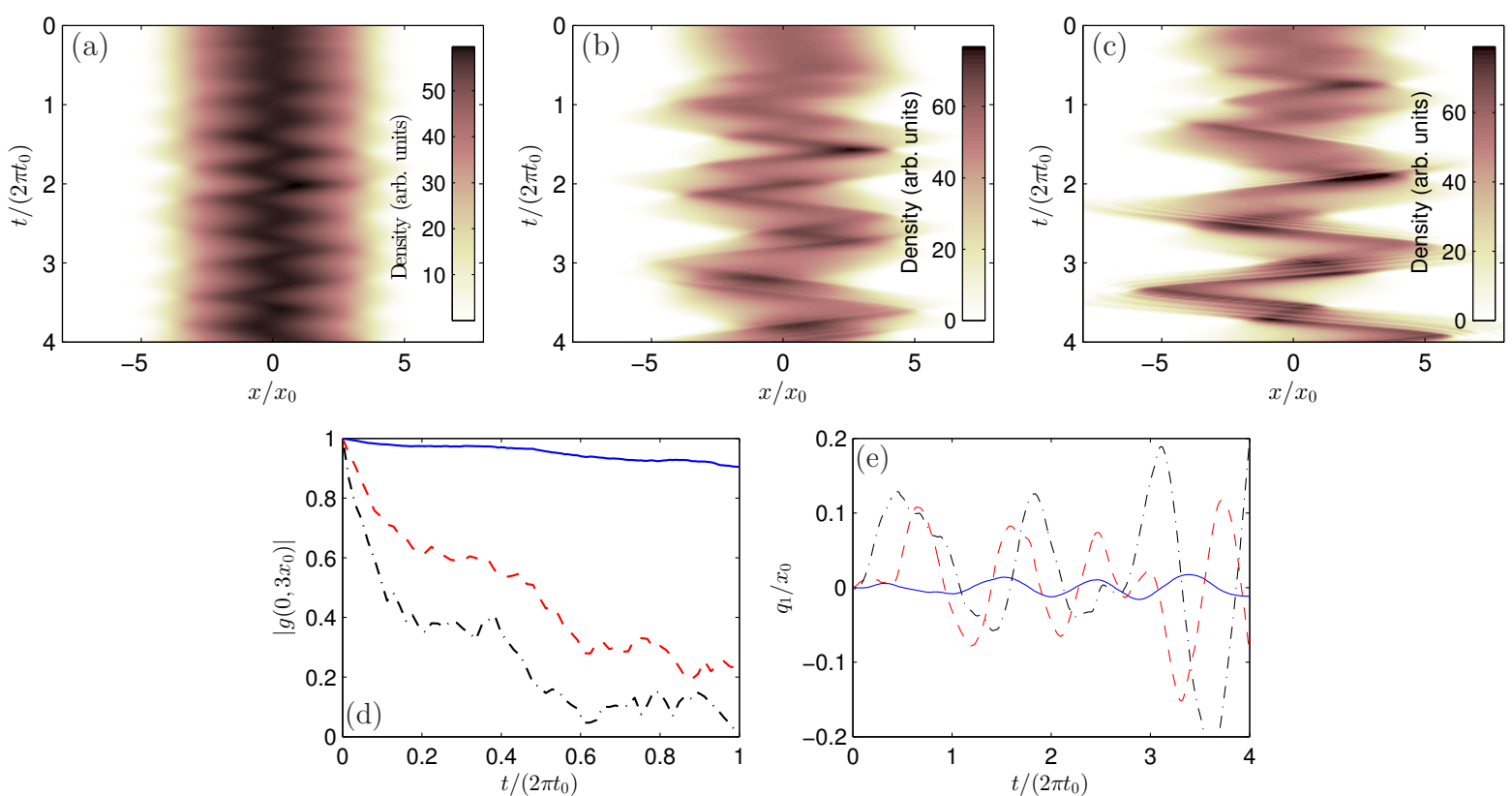

Figure 5. Effect of increasing transverse pump power $h_{0}$ while driving the Kohn mode. (a) Single realization of stochastic field density $|\psi(x, t)|^{2}$ evolution for pump power of $h_{0}=\bar{h}_{0}$ with $\bar{h}_{0}^{2} g_{0}^{2} / \kappa \Delta_{p a}^{2} \approx 0.042 \omega$ as was the case in Figure 2. (b) Single realization with $h_{0}=4 \bar{h}_{0}$. (c) Single realization with $h_{0}=6 \bar{h}_{0}$. (d) Short-time dissipation induced loss of coherence (ensemble averaged over 200 realizations) showing the increase in dissipation with pump power, with $h_{0}=\bar{h}_{0}$ (blue, solid), $h_{0}=4 \bar{h}_{0}$ (red, dashed) and $h_{0}=6 \bar{h}_{0}$ (black, dash-dotted).

(e) Center-of-mass displacement for the three trajectories shown in $(\mathbf{a}-\mathbf{c})$; line styles as in (d).

The measurement has a direct effect on the phase evolution of the atoms in such a way that the phase profile can considerably fluctuate between different individual runs. The spatially non-trivial dependence of these fluctuations results from the cavity field as well as from the transverse pump profile and detuning. No similar direct effect on the density profile exists and the density variation is 
a consequence of the phase dynamics. Averaging over many trajectories yields the loss of coherence, as also illustrated in Figure 5.

The spatially non-trivial dependence of fluctuations introduces a sensitivity of the measurements to the position of the atom cloud. In the limit of weak interactions, the atom cloud in the ground state would be approximated by the harmonic oscillator ground state wavefunction close to a potential minimum. In this limit the atoms would undergo zero point motion.

Owing to the multimode nature, the BEC exhibits several collective excitation modes that can be selectively driven. By altering the ratio of cavity wavelength to trap length, we can target different Bogoliubov collective modes. The next Bogoliubov mode with an odd symmetry is the one with the third lowest energy. Figure 6 shows the results with cavity wavenumber $k=1.03 x_{0}^{-1}$, chosen to predominantly excite this mode.
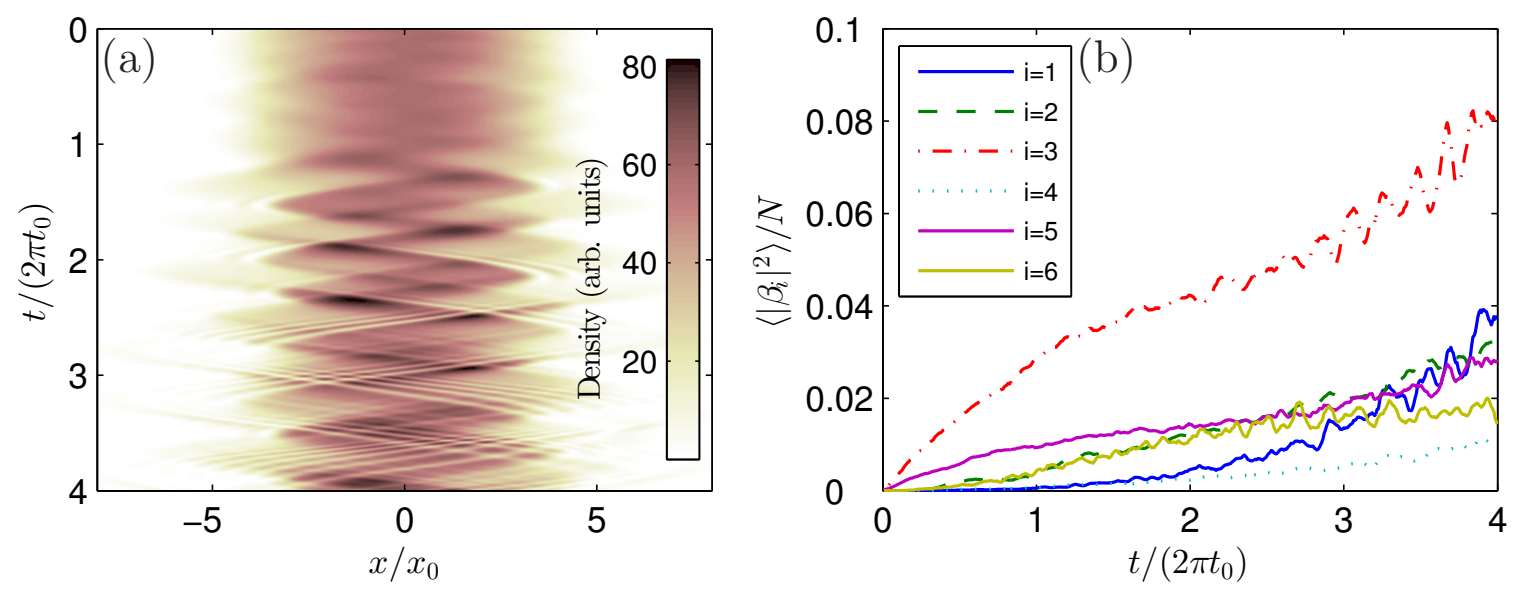

Figure 6. Response for a cavity wavelength of $k=1.03 x_{0}^{-1}$, such that the overlap integral $\mathrm{O}_{3}$ of Equation (43) provides maximal coupling to the third Bogoliubov mode. (a) Density of the stochastic field $|\psi(x, t)|^{2}$ for a single stochastic realization of a measurement record.

(b) Average single trajectory Bogoliubov mode populations, averaged over 400 realizations.

Subfigure (b) reproduced from [15].

By further changing the symmetry of $g(x)$ with respect to the trap center, which is by taking $g(x)=$ $g_{0} \cos (k x)$, we can selectively target modes of even symmetry. Figure 7 shows the targeted excitation of the lowest even mode, the breathing mode. Contrary to the case where odd modes are excited, the motion of an even symmetry mode does not change the symmetry properties of $\psi(x, t)$ with respect to the trap center, and consequently no odd modes are excited by this choice of $g(x)$. A possible practical problem for such cases may arise when the measured photon rate is considered. For even symmetry $g(x)$ the initial static condensate already generates a significant photon measurement rate, thus detection of oscillations in the photon measurement rate due to excitation of collective modes may be more difficult than in the odd symmetry case. However, in the case of the breathing mode, and for our choice of parameters, the signals in the photon measurement rate are clear. 

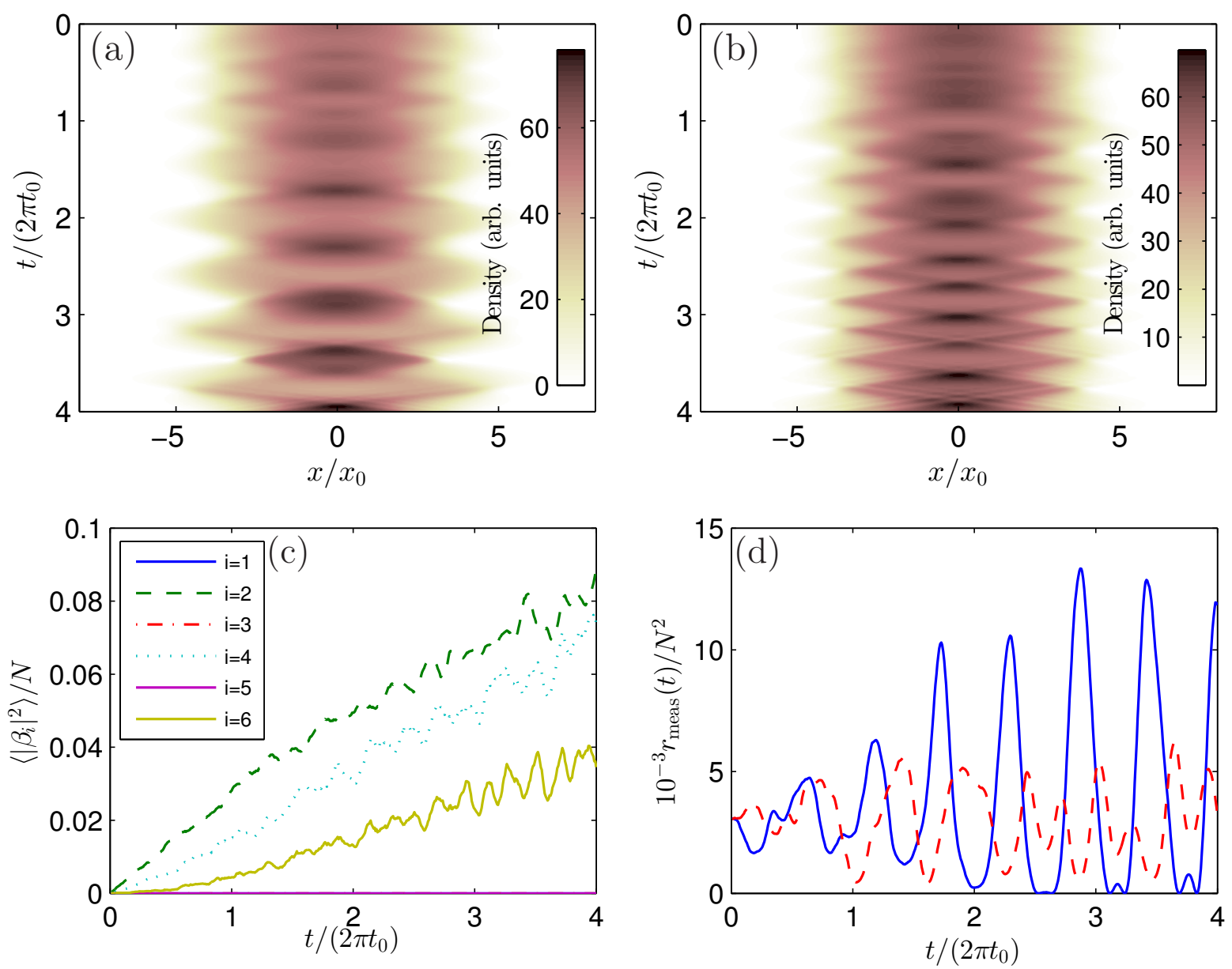

Figure 7. Response of the condensate to measurement backaction when the cavity mode coupling $g(x)$ is tailored to give a maximum overlap with the breathing mode, centering the trap potential on a cavity antinode such that $g(x)=g_{0} \cos (k x)$. (a,b) Two single realizations of the condensate stochastic field density $|\psi(x, t)|^{2}$. (c) Bogoliubov mode populations, averaged over 400 realizations. Subfigure reproduced from [15]. (d) Measurement rate $r_{\text {meas }}(t)$ corresponding to the density evolutions shown in (a) (blue, solid) and (b) (red, dashed).

\subsection{Phonon Detection}

In the previous section we discussed the selective excitation of phonons and the optical signatures of phonon modes when strong phonon excitations were present. It would be particularly promising to employ BEC-cavity systems for the detection of weakly excited phonon modes and even as potential single phonon detectors. Here we show that by counting photons outside the cavity that are almost solely scattered by the phonons we may accurately detect weak phonon excitations.

In order to analyze phonon modes quantum-mechanically, we take the Bogoliubov expansion Equation (33) and assume that only the BEC mode has a large occupation. Consequently, we replace $\hat{\beta}_{0} \sim \sqrt{N_{0}}$, where $N_{0}$ denotes the condensate particle number. For simplicity, in the following we also assume that the mode functions $\psi_{0}(x), u_{j}(x)$, and $v_{j}(x)$ are real. 
We further restrict the discussion to considering the detection of weak phonon excitations in a setup where the cavity is transversely pumped, such that the observable is $\hat{Y}$. However, the following treatment can be adapted to the cavity pumping case by a straightforward change to the operator $\hat{X}$. The light intensity measured outside the cavity consists of the coherently scattered part and the fluctuations $I_{\text {tot }}=I_{\text {coh }}+I_{\text {fl }}$. The coherent part $\propto|\langle\hat{a}\rangle|^{2}$ then depends on

$$
\langle\hat{a}\rangle=\frac{1}{\kappa}\langle\hat{Y}\rangle \simeq \frac{1}{\kappa} \int d x \frac{g(x) h(x)}{\Delta_{p a}}\left\{N_{0} \psi_{0}^{2}(x)+\sum_{j}\left[u_{j}^{2}(x) n_{j}+v_{j}^{2}(x)\left(n_{j}+1\right)\right]\right\} .
$$

The last expression with vanishing phonon excitations $\left(n_{j}=0\right)$ represents the condensate quantum depletion. For measurements of the light intensity fluctuations outside the cavity, we need to consider the observable

$$
\left\langle\hat{a}^{\dagger} \hat{a}\right\rangle-|\langle\hat{a}\rangle|^{2}=\frac{1}{\kappa^{2}}(\Delta \hat{Y})^{2},
$$

where

$$
(\Delta \hat{Y})^{2}=\left\langle\hat{Y}^{2}\right\rangle-\langle\hat{Y}\rangle^{2} \simeq N_{0} \sum_{j}\left\{\int d x \frac{g(x) h(x)}{\Delta_{p a}}\left[u_{j}(x)-v_{j}(x)\right] \psi_{0}(x)\right\}^{2}\left(2 n_{j}+1\right) .
$$

Here we have only kept the leading order contribution (for large $N_{0}$ ). The exact nature of the scattered intensity now depends on the choice of $g(x) h(x)$. For a system similar to that described in the previous section, with a uniform pump and a condensate trapped at a node of $g(x)$, then the symmetry of the system ensures that Equation (35) vanishes. Equation (37) is then the dominant term in the scattered light, and measurements of a given phonon mode can be made by maximizing the corresponding integral term. Figure 8 shows such overlaps for the odd numbered modes, while even mode overlaps vanish. The measurement rate of photons corresponding to Equation (37) does not need to be small and can also be considerably varied. For instance, for the parameters given in Section 3.1, for $N=1000$ we obtain the counting rate of about $10 \mathrm{\omega}$.

In general, the pump mode shape $h(x)$ can be tailored and the symmetry of the cavity mode can be altered, enabling phonon measurement either through maximizing Equation (37), as in Figure 8, or by minimizing Equation (37) such that the higher order terms dominate. The latter case is analogous to trying to influence Equation (35) such that the second term is comparable to the first term, and requires the integrals

$$
\int d x \frac{g(x) h(x)}{\Delta_{p a}} u_{j}^{2}(x), \quad \int d x \frac{g(x) h(x)}{\Delta_{p a}} v_{j}^{2}(x),
$$

to be maximized for some $j$ whilst simultaneously minimizing the overlap integral

$$
\int d x \frac{g(x) h(x)}{\Delta_{p a}} \psi_{0}^{2}(x) .
$$

The detection of quantum correlations of atoms confined in an optical cavity has previously been proposed, e.g., as an unambiguous signature of whether the atoms are in a Mott insulator or a superfluid state [59]. More generally, light can act as a sensitive diagnostic tool of ultracold atoms in periodic lattice systems, see (e.g., [60-65]). In addition, BEC-cavity systems have previously been considered, e.g., in weak force sensing applications $[66,67]$. 
Using the BEC-cavity system as a sensitive phonon detector could measure the statistical properties of phonons and therefore act as an accurate BEC thermometer or detect quantum correlations. The backaction of the quantum measurement process could be employed in preparation of complex quantum states of phonons. The phonon detector, based on the BEC-cavity system can also be considered as a counterpart to the idea to use a BEC as a single photon detector in a cavity $[68,69]$.

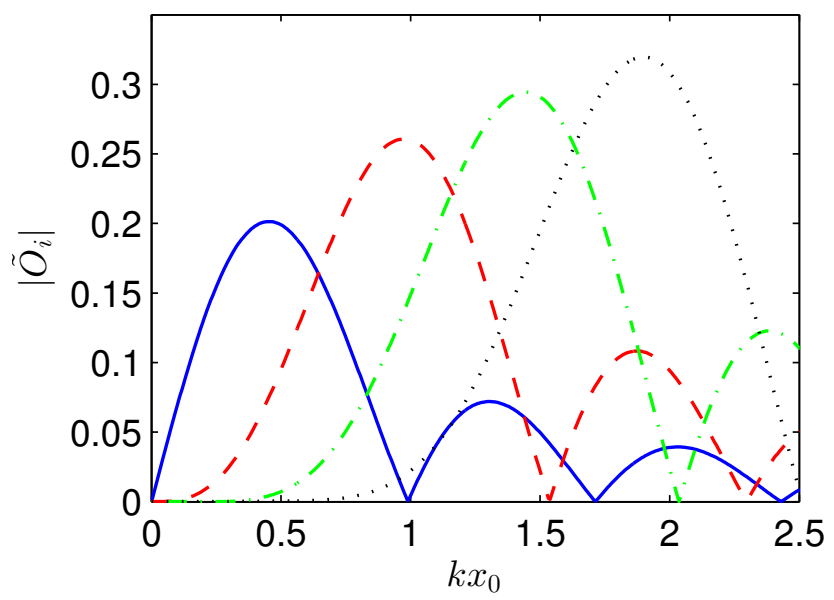

Figure 8. Overlap integral relevant to phonon detection in a transversely pumped cavity $\tilde{O}_{i}=1 /\left(g_{0} h_{0}\right) \int g(x) h(x)\left[u_{i}(x)-v_{i}(x)\right] \psi_{0}(x) d x$, as a function of the cavity wavenumber $k$, where $g(x)=g_{0} \sin (k x)$ and $h(x)=h_{0}$ is uniform. The condensate is centered at $x=0$, a node of the cavity light amplitude. We show such overlaps for the four lowest energy odd Bogoliubov modes $i=1$ (blue, solid), $i=3$ (red, dashed), $i=5$ (green, dot-dashed), and $i=7$ (black, dotted). Note that the symmetry of the system implies $\tilde{O}_{i}=0$ for even numbered modes, and the overlap with the condensate density of Equation (39) also vanishes in this system.

\section{Concluding Remarks}

Coupling interacting ultracold atoms to optical cavity fields provides a promising system to study several many-body realizations of quantum optical technologies, such as quantum state preparation and the effect of quantum measurement. Here we have investigated individual runs of continuously observed BECs in a cavity where the dynamics is conditioned on the measurement record. The method is based on classical measurement trajectories [15] that can numerically incorporate large many-atom systems with over a thousand spatial grid points by approximating the atom-cavity dynamics. Tailoring of the BEC-cavity system allows for the selective coupling of light to collective excitations of the condensate. In the weak excitation limit this could be applied to the development of a sensitive phonon detector.

In the ultracold many-atom context, the effect of quantum measurement and continuous monitoring of the dynamics have attracted considerable theoretical interest in the measurement schemes of atom counting [70-72], in the photon counting [73-76] and homodyne measurements [77] of scattered light, in dispersive imaging [45,78], and in an interferometric context [79]. Semiclassical [80] and static discrete approximations [81] have been considered for larger atom clouds in cavities. Feedback-control mechanisms [82] have also been employed in ultracold atom setups in approximate approaches [46]. 
In fact, simulations of the emergence of a relative phase between two BECs in a continuous quantum measurement process even when the BECs initially have no relative phase information [70-72,74] represent a measurement-induced spontaneous symmetry breaking analogous to that shown in our simulations (see Figure 3). In the phase measurements the phase is initially entirely random, but a continuous detection process eventually establishes a well-defined phase. Since in each simulation run this value emerges randomly, ensemble-averaging over many realizations results in a flat phase distribution $[0,2 \pi]$ and a fully restored symmetry, analogously to the restored symmetry of the density profile of Figure $3 b$.

\section{Acknowledgments}

This work was supported financially by the Engineering and Physical Sciences Research Council.

\section{Author Contributions}

Both authors contributed to the research and writing of the manuscript. The numerical simulations were performed by Mark D. Lee.

\section{Conflicts of Interest}

The authors declare no conflict of interest.

\section{Appendix}

Analyzing the Collective Mode Response

The excitation of different collective modes could be estimated in the limit of weak excitations by decomposing the BEC motion into the linearized Bogoliubov modes. Our treatment is related to the cavity cooling analysis in [17]. We use a classical version of the expansion Equation (33),

$$
\psi(x, t)=e^{-i \mu t / \hbar}\left\{\beta_{0}(t) \psi_{0}(x)+\sum_{i \neq 0}\left[\beta_{i}(t) u_{i}(x)-\beta_{i}^{*}(t) v_{i}^{*}(x)\right]\right\},
$$

where the quasiparticle modes $u_{i}(x)$ and $v_{i}(x)$ are the solutions to the Bogoliubov equations and the mode amplitudes $\beta_{j}$ are now treated classically. The expansion may be inverted to give the mode amplitudes as a function of the stochastic field from the classical measurement trajectories

$$
\beta_{i}=\int d x\left[u_{i}^{*}(x) \psi(x) e^{i \mu t / \hbar}+v_{i}^{*}(x) \psi^{*}(x) e^{-i \mu t / \hbar}\right] .
$$

The measurement of light intensity leaking out of the cavity represents the specific measurement of the atomic operator $\hat{Y}$, defined in Equation (14). We may now expand $\hat{Y}$ into the collective modes of the BEC inside the cavity

$$
\langle\hat{Y}\rangle=\frac{h_{0}}{\Delta_{p a}}\left\{\left|\beta_{0}\right|^{2} \int g(x)\left|\psi_{0}(x)\right|^{2} d x+\sum_{i \neq 0} \beta_{0}^{*} \int g(x) \psi_{0}^{*}(x)\left[\beta_{i} u_{i}(x)-\beta_{i}^{*} v_{i}(x)\right] d x\right\}
$$


where we have assumed $\left|\beta_{0}\right|^{2} \gg\left|\beta_{j}\right|^{2}$ for $j \neq 0$. We can therefore try to maximize the measurement backaction on specific collective mode $i$ by maximizing the corresponding overlap integral

$$
O_{i}=\int g(x) \psi_{0}^{*}(x)\left[u_{i}(x)-v_{i}(x)\right] d x
$$

\section{References}

1. Carmichael, H. Statistical Methods in Quantum Optics Vol 2; Springer: Berlin, Germany, 2007.

2. Brennecke, F.; Donner, T.; Ritter, S.; Bourdel, T.; Kohl, M.; Esslinger, T. Cavity QED with a Bose-Einstein condensate. Nature 2007, 450, 268-271.

3. Colombe, Y.; Steinmetz, T.; Dubois, G.; Linke, F.; Hunger, D.; Reichel, J. Strong atom-field coupling for Bose-Einstein condensates in an optical cavity on a chip. Nature 2007, 450, 272-276.

4. Murch, K.W.; Moore, K.L.; Gupta, S.; Stamper-Kurn, D.M. Observation of quantum-measurement backaction with an ultracold atomic gas. Nat. Phys. 2008, 4, 561-564.

5. Brahms, N.; Botter, T.; Schreppler, S.; Brooks, D.W.C.; Stamper-Kurn, D.M. Optical Detection of the Quantization of Collective Atomic Motion. Phys. Rev. Lett. 2012, doi:10.1103/PhysRevLett.108.133601.

6. Botter, T.; Brooks, D.W.C.; Schreppler, S.; Brahms, N.; Stamper-Kurn, D.M. Optical Readout of the Quantum Collective Motion of an Array of Atomic Ensembles. Phys. Rev. Lett. 2013, doi:10.1103/PhysRevLett.110.153001.

7. Schmidt, D.; Tomczyk, H.; Slama, S.; Zimmermann, C. Dynamical Instability of a Bose-Einstein Condensate in an Optical Ring Resonator. Phys. Rev. Lett. 2014, doi:10.1103/PhysRevLett.112.115302.

8. Kessler, H.; Klinder, J.; Wolke, M.; Hemmerich, A. Steering Matter Wave Superradiance with an Ultranarrow-Band Optical Cavity. Phys. Rev. Lett. 2014, doi:10.1103/PhysRevLett.113.070404.

9. Ritsch, H.; Domokos, P.; Brennecke, F.; Esslinger, T. Cold atoms in cavity-generated dynamical optical potentials. Rev. Mod. Phys. 2013, 85, 553-601.

10. Brennecke, F.; Ritter, S.; Donner, T.; Esslinger, T. Cavity Optomechanics with a Bose-Einstein Condensate. Science 2008, 322, 235-238.

11. Tian, L.; Carmichael, H.J. Quantum trajectory simulations of two-state behavior in an optical cavity containing one atom. Phys. Rev. A 1992, 46, R6801-R6804.

12. Dalibard, J.; Castin, Y.; Mølmer, K. Wave-function approach to dissipative processes in quantum optics. Phys. Rev. Lett. 1992, 68, 580-583.

13. Dum, R.; Zoller, P.; Ritsch, H. Monte Carlo simulation of the atomic master equation for spontaneous emission. Phys. Rev. A 1992, 45, 4879-4887.

14. Leroux, I.D.; Schleier-Smith, M.H.; Vuletić, V. Implementation of Cavity Squeezing of a Collective Atomic Spin. Phys. Rev. Lett. 2010, doi:10.1103/PhysRevLett.104.073602.

15. Lee, M.D.; Ruostekoski, J. Classical stochastic measurement trajectories: Bosonic atomic gases in an optical cavity and quantum measurement backaction. Phys. Rev. A 2014, doi:10.1103/PhysRevA.90.023628.

16. Javanainen, J.; Ruostekoski, J. Emergent classicality in continuous quantum measurements. New J. Phys. 2013, doi:10.1088/1367-2630/15/1/013005. 
17. Gardiner, S.A.; Gheri, K.M.; Zoller, P. Cavity-assisted quasiparticle damping in a Bose-Einstein condensate. Phys. Rev. A 2001, doi:10.1103/PhysRevA.63.051603.

18. Nagy, D.; Szirmai, G.; Domokos, P. Self-organization of a Bose-Einstein condensate in an optical cavity. Eur. Phys. J. D 2008, 48, 127-137.

19. Szirmai, G.; Nagy, D.; Domokos, P. Excess Noise Depletion of a Bose-Einstein Condensate in an Optical Cavity. Phys. Rev. Lett. 2009, doi:10.1103/PhysRevLett.102.080401.

20. Venkatesh, B.P.; O’Dell, D.H.J. Bloch oscillations of cold atoms in a cavity: Effects of quantum noise. Phys. Rev. A 2013, doi:10.1103/PhysRevA.88.013848.

21. Jaynes, E.; Cummings, F.W. Comparison of quantum and semiclassical radiation theories with application to the beam maser. Proc. IEEE 1963, 51, 89-109.

22. Walls, D.F.; Milburn, G.J. Quantum Optics, 2nd ed.; Springer: Berlin, Germany, 1994.

23. Maschler, C.; Mekhov, I.B.; Ritsch, H. Ultracold atoms in optical lattices generated by quantized light fields. Eur. Phys. J. D 2008, 46, 545-560.

24. Carmichael, H. An Open Systems Approach to Quantum Optics; Springer-Verlag: Berlin, Germany, 1993.

25. Gardiner, C.; Zoller, P. Quantum Noise; Springer: Berlin, Germany, 2004.

26. Olshanii, M. Atomic Scattering in the Presence of an External Confinement and a Gas of Impenetrable Bosons. Phys. Rev. Lett. 1998, 81, 938-941.

27. Ruostekoski, J.; Isella, L. Dissipative Quantum Dynamics of Bosonic Atoms in a Shallow 1D Optical Lattice. Phys. Rev. Lett. 2005, doi:10.1103/PhysRevLett.95.110403.

28. Carmichael, H. Statistical Methods in Quantum Optics Vol 1; Springer: Berlin, Germany, 1999.

29. Drummond, P.D.; Hardman, A.D. Simulation of Quantum Effects in Raman-Active Waveguides. Europhys. Lett. 1993, doi:10.1209/0295-5075/21/3/005.

30. Steel, M.J.; Olsen, M.K.; Plimak, L.I.; Drummond, P.D.; Tan, S.M.; Collett, M.J.; Walls, D.F.; Graham, R. Dynamical quantum noise in trapped Bose-Einstein condensates. Phys. Rev. A 1998, doi:10.1103/PhysRevA.58.4824.

31. Sinatra, A.; Lobo, C.; Castin, Y. The truncated Wigner method for Bose-condensed gases: Limits of validity and applications. J. Phys. B 2002, doi:10.1088/0953-4075/35/17/301.

32. Isella, L.; Ruostekoski, J. Quantum dynamics in splitting a harmonically trapped Bose-Einstein condensate by an optical lattice: Truncated Wigner approximation. Phys. Rev. A 2006, doi:10.1103/PhysRevA.74.063625.

33. Blakie, P.B.; Bradley, A.S.; Davis, M.J.; Ballagh, R.J.; Gardiner, C.W. Dynamics and statistical mechanics of ultra-cold Bose gases using c-field techniques. Adv. Phys. 2008, doi:10.1080/00018730802564254.

34. Martin, A.D.; Ruostekoski, J. Quantum and Thermal Effects of Dark Solitons in a One-Dimensional Bose Gas. Phys. Rev. Lett. 2010, doi:10.1103/PhysRevLett.104.194102.

35. Polkovnikov, A. Phase space representation of quantum dynamics. Ann. Phys. 2010, 325, 1790-1852.

36. Opanchuk, B.; Drummond, P.D. Functional Wigner representation of quantum dynamics of Bose-Einstein condensate. J. Math. Phys. 2013, doi:10.1063/1.4801781. 
37. Mathey, A.C.; Clark, C.W.; Mathey, L. Decay of a superfluid current of ultracold atoms in a toroidal trap. Phys. Rev. A 2014, doi:10.1103/PhysRevA.90.023604.

38. Gross, C.; Estève, J.; Oberthaler, M.K.; Martin, A.D.; Ruostekoski, J. Local and spatially extended sub-Poisson atom-number fluctuations in optical lattices. Phys. Rev. A 2011, doi:10.1103/PhysRevA.84.011609.

39. Plimak, L.I.; Olsen, M.K. Quantum-field-theoretical approach to phase-space techniques: Symmetric Wick theorem and multitime Wigner representation. Ann. Phys. 2014, 351, 593-619.

40. Martin, A.D.; Ruostekoski, J. Quantum dynamics of atomic bright solitons under splitting and recollision, and implications for interferometry. New J. Phys. 2012, doi:10.1088/1367-2630/14/4/043040.

41. Opanchuk, B.; Egorov, M.; Hoffmann, S.; Sidorov, A.I.; Drummond, P.D. Quantum noise in three-dimensional BEC interferometry. Europhys. Lett. 2012, doi:10.1209/0295-5075/97/50003.

42. Dujardin, J.; Uelles, A.A.; Schlagheck, P. Elastic and inelastic transmission in guided atom lasers: A truncated Wigner approach. Phy. Rev. A 2015, doi:10.1103/PhysRevA.91.033614.

43. Lewis-Swan, R.J.; Olsen, M.K.; Kheruntsyan, K.V. On the interpretation of single stochastic trajectories of the Wigner distribution. 2015, arXiv:1503.05647.

44. Norrie, A.A.; Ballagh, R.J.; Gardiner, C.W.; Bradley, A.S. Three-body recombination of ultracold Bose gases using the truncated Wigner method. Phys. Rev. A 2006, doi:10.1103/PhysRevA.73.043618.

45. Szigeti, S.S.; Hush, M.R.; Carvalho, A.R.R.; Hope, J.J. Continuous measurement feedback control of a Bose-Einstein condensate using phase-contrast imaging. Phys. Rev. A 2009, doi:10.1103/PhysRevA.80.013614.

46. Hush, M.R.; Szigeti, S.S.; Carvalho, A.R.R.; Hope, J.J. Controlling spontaneous-emission noise in measurement-based feedback cooling of a Bose-Einstein condensate. New J. Phys. 2013, doi:10.1088/1367-2630/15/11/113060.

47. Carmichael, H.J.; Nha, H. Vacuum fluctuations and the conditional homodyne detection of squeezed light. J. Opt. B Quant. Semiclass. Opt. 2004, doi:10.1088/1464-4266/6/8/004.

48. Ruostekoski, J.; Martin, A.D. Quantum Gases: Finite Temperature and Non-Equilibrium Dynamics, Cold Atoms Series; Proukakis, N.P., Gardiner, S.A., Eds.; Imperial College Press: London, UK, 2014.

49. Cattani, F.; Gross, C.; Oberthaler, M.K.; Ruostekoski, J. Measuring and engineering entropy and spin squeezing in weakly linked Bose-Einstein condensates. New J. Phys. 2013, doi:10.1088/1367-2630/15/6/063035.

50. Kippenberg, T.J.; Vahala, K.J. Cavity Opto-Mechanics. Opt. Express 2007, 15, 17172-17205.

51. Kippenberg, T.J.; Vahala, K.J. Cavity Optomechanics: Back-Action at the Mesoscale. Science 2008, 321, 1172-1176.

52. Meystre, P. A short walk through quantum optomechanics. Ann. Phys. 2013, 525, 215-233.

53. Aspelmeyer, M.; Kippenberg, T.J.; Marquardt, F. Cavity Optomechanics. 2013, arXiv:1303.0733.

54. O’Connell, A.D.; Hofheinz, M.; Ansmann, M.; Bialczak, R.C.; Lenander, M.; Lucero, E.; Neeley, M.; Sank, D.; Wang, H.; Weides, M.; et al. Quantum ground state and single-phonon control of a mechanical resonator. Nature 2010, 464, 697-703. 
55. Teufel, J.D.; Donner, T.; Li, D.; Harlow, J.W.; Allman, M.S.; Cicak, K.; Sirois, A.J.; Whittaker, J.D.; Lehnert, K.W.; Simmonds, R.W. Sideband cooling of micromechanical motion to the quantum ground state. Nature 2011, 475, 359-363.

56. Chan, J.; Alegre, T.P.M.; Safavi-Naeini, A.H.; Hill, J.T.; Krause, A.; Gröblacher, S.; Aspelmeyer, M.; Painter, O. Laser cooling of a nanomechanical oscillator into its quantum ground state. Nature 2011, 478, 89-92.

57. Brahms, N.; Stamper-Kurn, D.M. Spin optodynamics analog of cavity optomechanics. Phys. Rev. A 2010, doi:10.1103/PhysRevA.82.041804.

58. Gardiner, C. Stochastic Methods; Springer: Berlin, Germany, 2009.

59. Mekhov, I.B.; Maschler, C.; Ritsch, H. Cavity-Enhanced Light Scattering in Optical Lattices to Probe Atomic Quantum Statistics. Phys. Rev. Lett. 2007, doi:10.1103/PhysRevLett.98.100402.

60. Ruostekoski, J.; Foot, C.J.; Deb, A.B. Light Scattering for Thermometry of Fermionic Atoms in an Optical Lattice. Phys. Rev. Lett. 2009, doi:10.1103/PhysRevLett.103.170404.

61. Douglas, J.S.; Burnett, K. Imaging of quantum Hall states in ultracold atomic gases. Phys. Rev. A 2011, doi:10.1103/PhysRevA.84.053608.

62. Łakomy, K.; Idziaszek, Z.; Trippenbach, M. Thermal effects in light scattering from ultracold bosons in an optical lattice. Phys. Rev. A 2009, doi:10.1103/PhysRevA.80.043404.

63. Rist, S.; Menotti, C.; Morigi, G. Light scattering by ultracold atoms in an optical lattice. Phys. Rev. A 2010, doi:10.1103/PhysRevA.81.013404.

64. Corcovilos, T.A.; Baur, S.K.; Hitchcock, J.M.; Mueller, E.J.; Hulet, R.G. Detecting antiferromagnetism of atoms in an optical lattice via optical Bragg scattering. Phys. Rev. A 2010, doi:10.1103/PhysRevA.81.013415.

65. Cordobes Aguilar, F.; Ho, A.F.; Ruostekoski, J. Optical Signatures of Antiferromagnetic Ordering of Fermionic Atoms in an Optical Lattice. Phys. Rev. X 2014, doi:10.1103/PhysRevX.4.031036.

66. Goldwin, J.; Venkatesh, B.P.; O’Dell, D.H.J. Backaction-Driven Transport of Bloch Oscillating Atoms in Ring Cavities. Phys. Rev. Lett. 2014, doi:10.1103/PhysRevLett.113.073003.

67. Samoylova, M.; Piovella, N.; Robb, G.R.M.; Bachelard, R.; Courteille, P.W. Synchronization of Bloch oscillations by a ring cavity. Opt. Express 2015, 23, 14823-14835.

68. Horak, P.; Ritsch, H. Manipulating a Bose-Einstein condensate with a single photon. Eur. Phys. J. D Atom. Mol. Opt. Plasma Phys. 2001, 13, 279-287.

69. Horak, P.; Barnett, S.M.; Ritsch, H. Coherent dynamics of Bose-Einstein condensates in high-finesse optical cavities. Phys. Rev. A 2000, doi:10.1103/PhysRevA.61.033609.

70. Javanainen, J.; Yoo, S.M. Quantum Phase of a Bose-Einstein Condensate with an Arbitrary Number of Atoms. Phys. Rev. Lett. 1996, 76, 161-164.

71. Cirac, J.I.; Gardiner, C.W.; Naraschewski, M.; Zoller, P. Continuous observation of interference fringes from Bose condensates. Phys. Rev. A 1996, 54, R3714-R3717.

72. Castin, Y.; Dalibard, J. Relative phase of two Bose-Einstein condensates. Phys. Rev. A 1997, 55, 4330-4337.

73. Ruostekoski, J.; Walls, D.F. Bose-Einstein condensate in a double-well potential as an open quantum system. Phys. Rev. A 1998, 58, R50-R53. 
74. Ruostekoski, J.; Walls, D.F. Nondestructive optical measurement of relative phase between two Bose-Einstein condensates. Phys. Rev. A 1997, 56, 2996-3006.

75. Javanainen, J. Nonlinearity from quantum mechanics: Dynamically unstable Bose-Einstein condensate in a double-well trap. Phys. Rev. A 2010, doi:10.1103/PhysRevA.81.051602.

76. Elliott, T.J.; Kozlowski, W.; Caballero-Benitez, S.F.; Mekhov, I.B. Multipartite Entangled Spatial Modes of Ultracold Atoms Generated and Controlled by Quantum Measurement. Phys. Rev. Lett. 2015, doi:10.1103/PhysRevLett.114.113604.

77. Corney, J.F.; Milburn, G.J. Homodyne measurements on a Bose-Einstein condensate. Phys. Rev. A 1998, 58, 2399-2406.

78. Dalvit, D.A.R.; Dziarmaga, J.; Onofrio, R. Continuous quantum measurement of a Bose-Einstein condensate: A stochastic Gross-Pitaevskii equation. Phys. Rev. A 2002, doi:10.1103/PhysRevA.65.053604.

79. Lee, M.D.; Rist, S.; Ruostekoski, J. Bragg spectroscopic interferometer and quantum measurement-induced correlations in atomic Bose-Einstein condensates. New J. Phys. 2012, doi:10.1088/1367-2630/14/7/073057.

80. Niedenzu, W.; Schütz, S.; Habibian, H.; Morigi, G.; Ritsch, H. Seeding patterns for self-organization of photons and atoms. Phys. Rev. A 2013, doi:10.1103/PhysRevA.88.033830.

81. Mekhov, I.B.; Ritsch, H. Quantum Nondemolition Measurements and State Preparation in Quantum Gases by Light Detection. Phys. Rev. Lett. 2009, doi:10.1103/PhysRevLett.102.020403.

82. Wiseman, H.; Milburn, G. Quantum Measurement and Control; Cambridge University Press: Cambridge, UK, 2010.

(c) 2015 by the authors; licensee MDPI, Basel, Switzerland. This article is an open access article distributed under the terms and conditions of the Creative Commons Attribution license (http://creativecommons.org/licenses/by/4.0/). 